\title{
Grado de predicción de la motivación hacia las actividades físicodeportivas a través de la orientación de metas, la percepción del éxito y el grado de satisfacción. Un análisis transcultural*
}

\section{Prediction Level of Motivation through Physical and Sport Activities according to Goal Orientation, Success Perceived and Satisfaction Rate. A Cross-Cultural Analysis}

\author{
Luis A. Berlanga \\ Universidad Francisco de Vitoria, España \\ ORCID: http://orcid.org/0000-0002-0309-6568 \\ Constanza Palomino Devia \\ Universidad del Tolima, Colombia \\ Francisco Ruiz-Juan \\ Universidad de Murcia, España \\ Jose Antonio Gonzalez-Jurado \\ Universidad Pablo de Olavide, España
}

\footnotetext{
a Autor de correspondencia. Correo electrónico: luis.berlanga@ufv.es

Para citar este artículo: Berlanga, L. A., Palomino Devia, C., Ruiz-Juan, F., \& Gonzalez-Jurado, J. A. (2018). Grado de predicción de la motivación hacia las actividades físicodeportivas a través de la orientación de metas, la percepción del éxito y el grado de satisfacción. Un análisis transcultural. Universitas Psychologica, 17(1), 1-14. https://doi.org/10.11144/Ja veriana.upsy17-1.gpma
}

\section{RESUMEN}

El objetivo de este estudio fue conocer en qué medida se puede predecir el tipo de motivación hacia la práctica de actividad físicodeportiva en función de la orientación de metas, la percepción del éxito y el grado de satisfacción, mediante un análisis transcultural en tres países latinos: España, México y Costa Rica. Se evaluaron 2168 escolares de edades entre 11 y 16 años, utilizando cuatro instrumentos: Sport Motivation Scale (SMS), Task and Ego Orientation Sport Questionnaire (TEOSQ), Satisfaction Instrument (SSI) y Beliefs about the Causes of Sport Success Questionnaire (BACS). Los resultados mostraron que valores altos en orientación a la tarea, diversión y esfuerzo pueden predecir significativamente la manifestación de motivación intrínseca en los sujetos de los tres países.

Palabras clave

motivación; satisfacción; éxito; orientación de metas; actividades físicas. 
Results showed that high values in task orientation, fun, and effort could significantly predict intrinsic motivation in the three countries of our research.

Keywords

motivation; satisfaction; success; goal orientation; physical activity.

La participación de niños y niñas en actividades físicodeportivas (AFD) de forma organizada y sistemática está considerada como una oportunidad clave para el desarrollo de las habilidades motrices, sociales, el autoestima y el mantenimiento de la salud (Zaff, Moore, Papillo, \& Williams, 2003). Además, en países occidentales, está comúnmente aceptada como parte del desarrollo infantil (Salguero, GonzalezBoto, Tuero, \& Marquez, 2003), por lo que la motivación de los niños para hacer parte en este tipo de programas es de gran importancia para los investigadores, entrenadores, profesores y padres (Keegan, Harwood, Spray, \& Lavallee, 2009).

En este sentido, se debe tener en cuenta que una simple descripción de los motivos que inducen a la práctica de estas actividades no necesariamente puede ofrecer información sobre cómo los jóvenes aprecian sus experiencias en actividades deportivas, sino que es necesaria una mayor exploración del concepto de motivación (Franco, Coteron, Gómez, Brito, \& Martinez, 2017; Morgan, 2017; Zahariadis \& Biddle, 2000). Por consiguiente, para una adecuada justificación del objeto de investigación de este estudio, se han considerado las teorías que han analizado la motivación en el comportamiento humano.

Tras estudiar a diferentes autores que han llevado a cabo sus investigaciones en este ámbito (Locke \& Bryan, 1969; Nicholls, 1989; Vroom, 1964), podríamos definir la motivación como un conjunto de procesos que determinan el inicio, mantenimiento y finalización de cualquier conducta. Se ha definido la motivación como el deseo de hacer un gran esfuerzo por alcanzar alguna meta, estando dicho deseo condicionado por la necesidad de satisfacer alguna necesidad individual (Robbins \& Judge, 2009). Atendiendo a estas y otras definiciones de diversos autores, se puede observar como la motivación es el determinante inmediato y más importante del comportamiento humano (Böckler, Tusche, \& Singer, 2016; Murray, 1964; O’Doherty, 2016; Roberts, 2012).

Muchas investigaciones sobre la motivación en la práctica de AFD se han centrado, principalmente, en el estudio de la autodeterminación (Brooks et al., 2017; Deci \& Ryan, 2000; Fletcher, 2016) y de las orientaciones de metas (Duda \& Whitehead, 1998), siendo esta última una de las teorías de la motivación más populares y mejor investigadas que sirve para analizar la motivación y la búsqueda de éxito en dicha práctica (Abraldes, Granero-Gallegos, Baena-Extremera, Gómez-López, \& RodríguezSuárez, 2016; da Costa, Hirota, \& de Marco, 2015; Grastén, 2016; Jaakkola, Ntoumanis, \& Liukkonen, 2016).

La teoría de las orientaciones de metas, enunciada por Nicholls (1989), articula dos orientaciones predominantes de involucrarse para alcanzar un éxito, denominadas orientación al ego y orientación a la tarea, que sirven como antecedentes claves para detectar diferencias tanto en la motivación comportamental de los sujetos como en sus respuestas cognitivas y afectivas. Se ha informado que deportistas que presentan altos niveles de orientación a la tarea suelen mostrar mayores niveles de disfrute con la práctica de AFD y son más propensos a ser constantes y esforzarse ante tareas complejas (Gutiérrez, Tomás, \& Calatayud, 2017). Por otro lado, aquellos con altos niveles de orientación al ego exhiben un perfil psicológico y comportamental menos positivo ante la práctica de estas actividades, aunque ambos pueden demostrar altos niveles de rendimiento deportivo (Smith, Cumming, \& Smoll, 2008).

Con respecto a la teoría de la autodeterminación (Deci \& Ryan, 2000), se podría definir como una macroteoría de la motivación humana que tiene relación con el desarrollo y el funcionamiento de la personalidad dentro de los contextos sociales (MorenoMurcia \& Martínez, 2006). La teoría de la autodeterminación (TAD) supone un enfoque hacia la motivación humana y la personalidad encargadas de la autorregulación de la conducta (Deci \& Ryan, 1985), lo cual representa el grado 
en que las personas realizan sus acciones de forma voluntaria y por su propia elección. La TAD establece diferentes tipos de motivación que se dan siguiendo un continuum (Deci \& Ryan, 1985, 2000; Ryan \& Deci, 2000). La mayoría de las investigaciones en el ámbito de la motivación tienden a indicar que los tipos de motivación más autodeterminada, como la motivación intrínseca y la regulación identificada, están conectados con consecuencias positivas (Menéndez Santurio \& Fernández-Río, 2017; Vallerand, 2012; Vink, Raudsepp, \& Kais, 2015).

En el ámbito de la práctica de AFD y otros ámbitos del comportamiento humano, es importante tener presentes las influencias de los contextos culturales sobre la muestra que se analiza. Aunque muchos de los motivos que inducen a la práctica de AFD son comunes en la mayoría de las culturas, se han reportado diferencias que vislumbran la necesidad de desarrollar análisis transculturales, de modo que se pueda determinar si las variables sociales y cognitivas que se analizan tienen $\mathrm{o}$ no una importancia universal (Franco, Coterón, Martínez, \& Brito, 2017; Han, Mahony, \& Greenwell, 2016; Keegan, Middleton, Henderson, \& Girling, 2016; Kouli \& Papaioannou, 2009; Nishida, Isogai, Åström, Karp, \& Johansson, 2007; Vilchez Conesa \& Ruiz Juan, 2016).

Así, el objetivo de nuestro estudio es analizar en qué medida se puede predecir el tipo de motivación hacia la práctica de AFD en función de la orientación de metas, del grado de satisfacción y de la percepción del éxito en un análisis transcultural en tres países latinos: España, México y Costa Rica.

\section{Método}

\section{Participantes}

Participaron 2168 estudiantes del primer curso de enseñanza secundaria, seleccionados aleatoriamente, procedentes de Costa Rica (423), México (408) y España (1337), siendo 1052 hombres (50.4\%), 1037 mujeres (49.6\%) y
79 no consignaron el sexo, y de centros públicos (86.6 \%) y concertados (13.4\%). El rango de edad estuvo entre 11 y 16 años $(M=12.49 ; D E$ $=0.81$ ), siendo la edad media en hombres 12.53 $(D E=0.87)$ y $12.44(D E=0.74)$ en mujeres.

\section{Diseño}

Se llevó a cabo una investigación descriptiva e inferencial para dar respuesta a los objetivos planteados en la misma.

\section{Procedimiento}

Se solicitó autorización por escrito a los centros educativos explicando detalladamente los objetivos de la investigación y su procedimiento y acompañando un modelo del instrumento que se iba a utilizar. La prueba fue autoadministrada, con aplicación masiva y la participación fue voluntaria y totalmente anónima. Los sujetos fueron informados del objetivo del estudio, se les indicó que no había respuestas correctas o incorrectas, solicitándoles la máxima sinceridad y honestidad. Solamente los alumnos que contaban con consentimiento informado de los tutores legales participaron en la investigación.

Instrumentos y variables de estudio

Escala de Motivación Deportiva [Sport Motivation Scale (SMS)] (Pelletier, Fortier, Vallerand, Eु Tuson, 1995)

Se aplicó una versión validada para español (Núñez, Martín-Albo, Navarro, \& González, 2006). Fue elaborada para medir tres tipos de motivación intrínseca (al conocimiento, al logro y a las experiencias estimulantes), tres tipos de motivación extrínseca (regulación externa, regulación introyectada y regulación identificada) y la amotivación. Consta de 28 ítems distribuidos en siete subescalas, correspondientes a los siete tipos de motivación mencionados, con cuatro ítems cada escala, 
puntuando en escala tipo Likert de 7 puntos desde (1) No se corresponde en absoluto, hasta (7) Se corresponde totalmente. Este instrumento permite calcular el índice de autodeterminación (SDI), combinando las diferentes subescalas y calculándolo con la ecuación que se observa en la figura 1.

\section{Figura 1}

Ecuación para calcular el indice de autodeterminación (SDI).

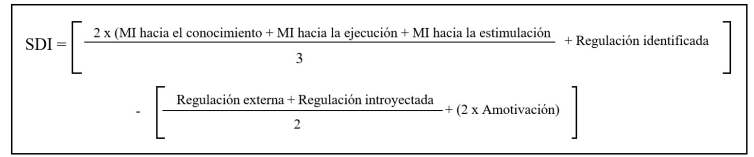

Fuentes: Vallerand (1997);

Vallerand y Rousseau (2001).

Cuestionario de Orientación al Ego y a la Tarea en el Deporte [Task and Ego Orientation Sport Questionnaire (TEOSQ)] (Balaguer, Castillo, $\mathfrak{E}$ Tomás, 1996; Duda, 1989)

El cuestionario valora la tendencia hacia una orientación de meta al ego y a la tarea en el contexto deportivo, mediante 13 ítems divididos en dos escalas que miden orientación tarea (7 ítems) y orientación ego (6 ítems). En las instrucciones se pide a los sujetos que piensen cuando se sienten con más éxito en su deporte, indicando el grado de acuerdo con los ítems con respuestas en escala tipo Likert que oscila desde (1) muy en desacuerdo a (5) muy de acuerdo.

Cuestionario de Satisfacción Intrínseca en el Deporte (Balaguer, Atienza, Castillo, MorenoMurcia, $\mathcal{E}$ Duda, 1997) [versión en español del Sport Satisfaction Instrument (SSI) de Duda $\mathbb{E}$ Nicholls, 1992]

Elaborado para determinar el grado de divertimento cuando se realiza actividad físicodeportiva en tiempo libre. Consta de 8 ítems divididos en dos escalas que miden diversión (5 ítems) y aburrimiento (3 ítems) en la práctica deportiva. Los sujetos deben indicar su grado de acuerdo con los ítems, recogiéndose las respuestas en escala Likert que oscila desde 1 (muy en desacuerdo) a 5 (muy de acuerdo).

Inventario de Percepción de las Creencias sobre las Causas del Éxito en el Deporte (Castillo, Balaguer, Eु Duda, 2002) [versión española del Beliefs About the Causes of Sport Success Questionnaire (BACSSQ) de Duda Eु Nicholls, 1992)

Consta de 18 ítems que miden las percepciones que tienen los sujetos sobre si el esfuerzo ( 9 ítems, esfuerzo ejercido en el desempeño de la tarea), la habilidad (4 ítems, factores relacionados con la posesión de habilidad) y el uso de técnicas de engaño (5 ítems, utilizar conductas engañosas) permiten alcanzar el éxito en el deporte. En las instrucciones se pregunta a los sujetos: "¿Qué crees que debería hacer la gente para tener éxito en el deporte que practica más a menudo?", teniendo esa pregunta en cuenta, ellos deben responder a cada ítem que se presenta utilizando una escala tipo Likert desde (1) muy en desacuerdo a (5) muy de acuerdo.

\section{Análisis de los datos}

Se llevaron a cabo análisis de ítems, homogeneidad entre ítems, correlación entre las subescalas (coeficiente de Pearson), consistencia interna (alfa de Cronbach), diferencias de medias por países (ANOVA) y la prueba post-hoc (Scheffé). Se llevó a cabo una regresión lineal multivariante, de la cual se obtuvo el valor de R2 corregida para explicar el nivel de varianza del modelo regresión completo, el coeficiente Beta para explicar la predicción entre cada una de las variables del modelo, el estadístico $F$ para comprobar si existe relación entre las variables seleccionadas y su significatividad estadística. Se asumió la significación estadística para un valor de $p \leq 0.05$. El tratamiento estadístico de los datos se realizó con el software IBM-SPSS 22. La estructura factorial se examinó con un Análisis Factorial Confirmatorio (AFC) con el software AMOS 21.0. 


\section{Resultados}

Para calcular las propiedades psicométricas se siguió el procedimiento de análisis establecido por Carretero-Dios y Pérez (2005) . En el análisis de ítems en las cuatro escalas, ningún ítem fue eliminado al cumplir los requisitos establecidos (valor $\geq 0.3$ en el coeficiente de correlación corregido ítem-total, desviación estándar $>1$ y se usaron todas las opciones de respuesta). El análisis de la homogeneidad indicó la inexistencia de solapamientos de ítems entre las dimensiones teóricas utilizadas en cada una de las cuatro escalas. Se calcularon los índices de asimetría y curtosis siendo, en general, próximos a cero y $<2$, tal y como recomiendan Bollen y Long (1994).

\section{Tabla 1}

Índices de ajuste del modelo

\begin{tabular}{|c|c|c|c|c|c|c|c|c|}
\hline Pais & Escala & $n$ & $x^{2 / g l}$ & TLI & IFI & CFI & RMSEA & SRMR \\
\hline \multirow{4}{*}{ Costa Rica } & $\begin{array}{l}\text { Motivación en la práctica fisicodeportiva } \\
\text { (SMS) }\end{array}$ & 298 & 2.18 & 0.95 & 0.94 & 0.93 & 0.03 & 0.02 \\
\hline & Orientaciones de meta (TEOSQ) & 288 & 2.38 & 0.96 & 0.96 & 0.95 & 0.03 & 0.02 \\
\hline & $\begin{array}{l}\text { Satisfacción intrínseca en el deporte } \\
\text { (SSI) }\end{array}$ & 311 & 2.59 & 0.93 & 0.93 & 0.93 & 0.04 & 0.03 \\
\hline & $\begin{array}{l}\text { Percepción de las creencias sobre las } \\
\text { causas del éxito en el deporte (BACS) }\end{array}$ & 313 & 3.12 & 0.92 & 0.92 & 0.91 & 0.04 & 0.04 \\
\hline \multirow{4}{*}{ México } & $\begin{array}{l}\text { Motivación en la práctica físico- } \\
\text { deportiva (SMS) }\end{array}$ & 137 & 3.57 & 0.92 & 0.91 & 0.92 & 0.06 & 0.04 \\
\hline & Orientaciones de meta (TEOSQ) & 137 & 2.85 & 0.93 & 0.94 & 0.93 & 0.04 & 0.04 \\
\hline & $\begin{array}{l}\text { Satisfacción intrínseca en el deporte } \\
\text { (SSI) }\end{array}$ & 137 & 3.75 & 0.94 & 0.92 & 0.93 & 0.06 & 0.03 \\
\hline & $\begin{array}{l}\text { Percepción de las creencias sobre las } \\
\text { causas del éxito en el deporte (BACS) }\end{array}$ & 394 & 3.43 & 0.95 & 0.93 & 0.95 & 0.06 & 0.03 \\
\hline \multirow{5}{*}{ España } & $\begin{array}{l}\text { Motivación en la práctica fisico- } \\
\text { deportiva (SMS) }\end{array}$ & 798 & 3.17 & 0.94 & 0.94 & 0.94 & 0.06 & 0.04 \\
\hline & Orientaciones de meta (TEOSQ) & 789 & 2.98 & 0.96 & 0.96 & 0.95 & 0.04 & 0.03 \\
\hline & $\begin{array}{l}\text { Satisfacción intrínseca en el deporte } \\
\text { (SSI) }\end{array}$ & 812 & 3.72 & 0.95 & 0.94 & 0.95 & 0.06 & 0.03 \\
\hline & $\begin{array}{l}\text { Percepción de las creencias sobre las } \\
\text { causas del éxito en el deporte (BACS) }\end{array}$ & 1061 & 3.11 & 0.95 & 0.93 & 0.95 & 0.06 & 3 \\
\hline & Deseable & & $<5$ & $>0.9$ & $>0.9$ & $>0.9$ & $<0.08$ & $<0.05$ \\
\hline
\end{tabular}

$\chi 2 / g 1:$ La razón entre chi-cuadrado y el número de grados de libertad; SRMR: Raíz del residuo cuadrático estandarizado; RMSEA:

Raíz del residuo cuadrático promedio de aproximación; TLI: Índice de Tucker-

Lewis; CFI: Índice de bondad de ajuste comparativo; IFI: Índice de ajuste incremental.

La validez factorial de los cuatro instrumentos ha sido examinada usando análisis factorial confirmatorio (AFC). Al no existir normalidad multivariada en todas las variables estudiadas, se utilizó estimación de máxima verosimilitud y se tuvo que recurrir al método bootstrapping, procedimiento de estimación de modelos de ecuaciones estructurales que asume distribución normal univariada. El ajuste del modelo fue evaluado con combinación de índices de ajuste absolutos y relativos. Los modelos de las cuatro escalas presentan valores correctos que permiten determinar una aceptable bondad de ajuste del modelo original (Hoyle, 1995; Hu \& Bentler, 1999; Kline, 1998) manifestado por los resultados obtenidos (Tabla 1).

En la Tabla 2 se presentan los coeficientes alfa de Cronbach y los promedios y desviaciones estándar de las diferentes escalas y dimensiones. Todas las escalas y subescalas demostraron una consistencia interna satisfactoria (entre $a=$ 0.7 y $a=0.94)$. Asimismo, existen diferencias estadísticamente significativas $(p<0.001)$ entre las medias de cada una de las variables analizadas por países.

En la motivación, el alumnado mexicano presenta los promedios más altos en cada una de las variables. Se aprecia una tendencia de incremento importante de las medias a medida que se incrementa el nivel de autodeterminación, pasando en los mexicanos de valores de $M=4.02$ $(D E=1.63)$ en amotivación a $M=5.63(D E=$ 1.28) en motivación intrínseca de conocimiento. Los españoles son los que presentan menor amotivación $(M=2.83, D E=1.6)$. Sin embargo, el índice de autodeterminación (SDI) es mayor en los españoles $(M=4.6, D E=4.44)$, seguido de mexicanos $(M=2.91, D E=3.83)$ y costarricenses $(M=2.58, D E=4.32)$. La prueba post-hoc de Scheffé indica, en todas las variables, la existencia de tres subconjuntos homogéneos correspondientes a cada país.

Con respecto a las orientaciones de meta, se aprecia como las mayores puntuaciones se dan en orientación a la tarea y las más bajas en orientación al ego en los tres países. La prueba post-hoc de Scheffé indica, en las dos variables, la existencia de tres subconjuntos homogéneos correspondientes a cada país. La satisfacción intrínseca en el deporte presenta medias altas en la variable diversión en los tres países, estando el alumnado español y mexicano $(M=4.24, D E=$ $0.82 ; M=4.23, D E=0.85$, respectivamente) ligeramente por encima del costarricense $(\mathrm{M}=$ $3.95, D E=1)$. Sin embargo, en la variable aburrimiento los valores medios son bajos, siendo los españoles y mexicanos quienes presentan la 
menor media $(M=1.93, D E=0.89 ; M=2, D E$ $=1.07)$. En ambas variables, la prueba post-hoc de Scheffé indica la existencia de dos subconjuntos homogéneos correspondiente uno a Costa Rica y el otro a España y México.

En la percepción de las creencias sobre las causas del éxito en el deporte se encuentran diferencias estadísticamente significativas en las tres variables. En la variable esfuerzo, es el alumnado mexicano el que presenta los valores más elevados $(M=4.01, D E=0.83)$, seguido del español $(M=3.81, D E=0.88)$ y del costarricense $(M=3.39, D E=0.98)$. Pero siempre con valores superiores a la creencia de la habilidad (medias entre 3.61 y 2.8) y del engaño (medias entre 3.02 y 2.4). La prueba post-hoc de Scheffé indica, en las tres variables, la existencia de tres subconjuntos homogéneos correspondientes a cada país.

\section{Tabla 2}

Coeficiente alfa, media (M) y desviación estándar (DE) de las escalas de motivación aplicadas. Diferencias por países

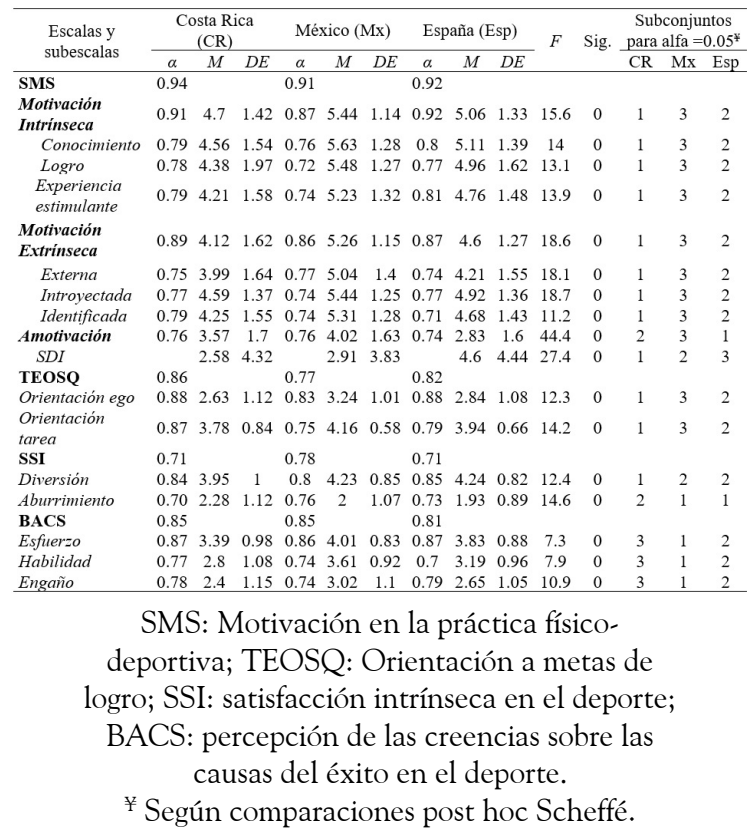

En la Tabla 3, quedan reflejados los resultados de las correlaciones que se calcularon. En la motivación intrínseca, en los tres países, se obtuvo una correlación significativa y positiva con motivación extrínseca, orientación al ego, orientación a la tarea, diversión, esfuerzo y habilidad; en España, además correlaciona negativamente con aburrimiento.

En la motivación extrínseca se obtuvieron correlaciones positivas y significativas con motivación intrínseca, amotivación, orientación al ego, orientación a la tarea, diversión, esfuerzo, habilidad y engaño, en los tres países.

En la amotivación se obtuvo, en los tres países, una correlación positiva y significativa con motivación extrínseca, orientación al ego, aburrimiento, habilidad y engaño, también correlaciona negativamente con esfuerzo y, en España, además con diversión.

En el índice de autodeterminación (SDI), las correlaciones significativas y positivas, en los tres países, fueron con orientación a la tarea, diversión (salvo en México) y esfuerzo. Las correlaciones significativas y negativas fueron con orientación al ego (salvo en México), aburrimiento y engaño.

\section{Tabla 3}

Correlaciones entre las subescalas de cada instrumento aplicado y las dimensiones de Escala de Motivación en la Práctica Físico-deportiva (SMS).

Diferencias por países

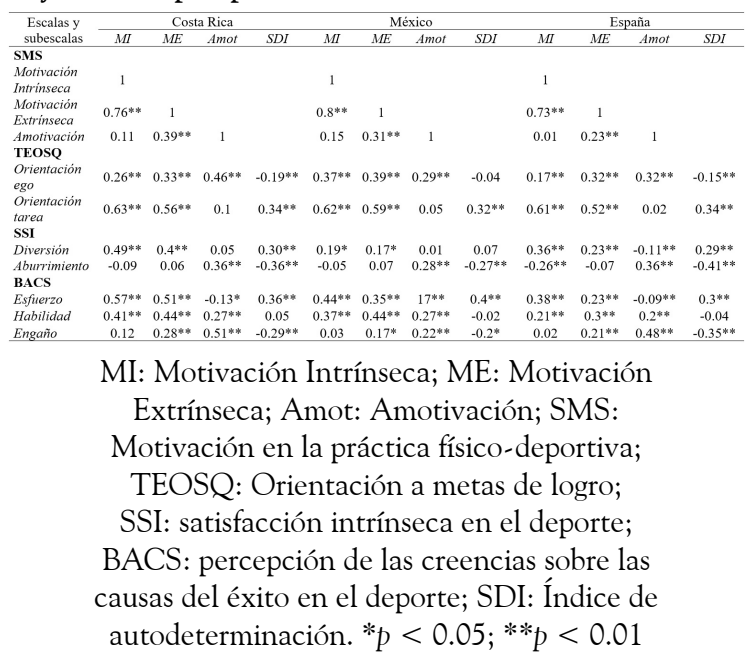


Tabla 4

Análisis de Regresión Multivariante. Modelos que predicen significativamente la Motivación en la Práctica Físico-deportiva (SMS), por países

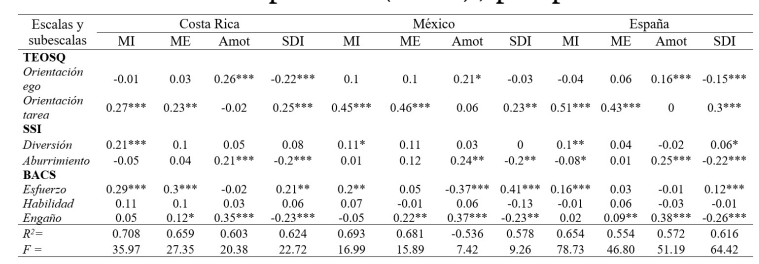

MI: Motivación Intrínseca; ME:

Motivación Extrínseca; Amot: Amotivación; SMS: Motivación en la práctica físicodeportiva; TEOSQ: Orientación a metas de logro; SSI: satisfacción intrínseca en el deporte; BACS: percepción de las creencias sobre las causas del éxito en el deporte; SDI: Índice de autodeterminación. Los valores están dados en $\beta . * p<0.05 ; * p<0.01 ; * * p<0.001$

Se realizó un análisis de regresión lineal multivariado, tratando de obtener unos modelos que explicasen la mayor parte posible de varianza. Se tomaron como variables dependientes la puntuación media de cada una de las motivaciones de la SMS (motivación intrínseca, motivación extrínseca, amotivación e índice de autodeterminación) y como variables predictoras cada una de las variables de la orientación de meta en el deporte (orientación al ego y orientación a la tarea), la satisfacción intrínseca en el deporte (diversión y aburrimiento) y percepción de las creencias sobre las causas del éxito en el deporte (esfuerzo, habilidad y técnicas de engaño). Por último, como variable de selección se consideró el país.

Los modelos presentados explican más de la mitad de la varianza en los tres países, oscilando entre el $53.6 \%$ y el $70.8 \%$. Del análisis se extrajo el valor de $R^{2}$ para explicar la varianza, el coeficiente Beta para explicar la predicción entre variables y el de $F$ para constatar si existe relación entre las variables seleccionadas y su significatividad (Tabla 4).

El modelo obtenido muestra que la motivación intrínseca se puede predecir significativamente por puntuar alto en orientación a la tarea, diversión y esfuerzo, en los tres países, y bajo en aburrimiento en España (varianza: $70.8 \%$ Costa Rica, 69.3 \% México y 65.4 \% España).
El modelo obtenido de la motivación extrínseca muestra que se puede predecir significativamente por puntuar alto en orientación a la tarea y uso de técnicas de engaño, en los tres países, y esfuerzo en Costa Rica (varianza: 65.9 \% Costa Rica, 68.1 \% México y 55.4 \% España). El modelo obtenido de la amotivación, en los tres países, indica que se puede predecir significativamente por puntuar alto en orientación al ego, aburrimiento y uso de técnicas de engaño (varianza: 60.3 \% Costa Rica, 53.6 \% México y $57.2 \%$ España). El modelo obtenido del índice de autodeterminación (SDI), en los tres países, muestra que se puede predecir significativamente por puntuar alto en orientación a la tarea y esfuerzo y bajo en orientación al ego (salvo en México), aburrimiento y uso de técnicas de engaño (Tabla 4).

\section{Discusión}

El objetivo de la presente investigación fue analizar el grado de predicción de la motivación hacia la práctica de AFD con respecto a la orientación de metas, el grado de satisfacción y la percepción de las causas del éxito en un análisis transcultural en tres países latinos: España, México y Costa Rica.

Los resultados de nuestro estudio muestran medias más altas en motivación intrínseca para los tres países analizados. En las comparaciones entre países, se hallaron diferencias significativas de cada país respecto de los otros dos, si bien los estudiantes mexicanos superaron a españoles y costarricenses en todos los tipos de motivación, tanto a nivel intrínseco (conocimiento, logro y experiencias estimulantes) como extrínseco (externa, introyectada e identificada), incluso en amotivación (Tabla 2). Estos valores elevados de motivación entre los diferentes tipos están en consonancia con el estudio de Stuntz y Weiss (2009) , en el que los autores afirmaron que la forma en la que el entrenador o monitor deportivo interactúa con sus deportistas está relacionada con la falta de criterio para definir claramente cuáles son los motivos que les inducen a practicar AFD. Asimismo, conlleva 
una percepción de éxito en el deporte definido por diversas causas no claramente establecidas (Stuntz \& Weiss, 2009); lo cual también se demostró en nuestro estudio con valores elevados de los estudiantes mexicanos en la escala BACS en los tres parámetros que analiza (esfuerzo, habilidad y engaño), encontrando diferencias significativas en las tres variables al comparar los resultados entre países.

Kim, Williams y Gill (2003) llevaron a cabo estudios transculturales que demuestran diferentes tipos de motivación hacia la práctica de AFD entre países distintos. Así, estos autores demostraron que estudiantes de secundaria de Estados Unidos y de Corea presentan tipos de motivación diferentes hacia la práctica de AFD, siendo los americanos los que mostraron mayores niveles de motivación intrínseca con respecto a los coreanos. Otro estudio transcultural encontró también diferencias entre la motivación que los jóvenes practicantes de AFD presentaban, estudiada y comparada entre una población de Estados Unidos, otra de China y otra chinanorteamericana, donde los norteamericanos resultaron estar principalmente motivados hacia la competición y la necesidad de mejorar, los chinos se involucraban en estas actividades por motivos de afiliación social y de bienestar, y los chinos-norteamericanos participaban por el gusto de viajar, usar indumentaria oficial y divertirse (Yan \& McCullagh, 2004). Por ello, principalmente la similitud de resultados en cuanto a los niveles altos de motivación intrínseca en los tres países objeto de este estudio suponen un avance en el análisis transcultural de la motivación en el ámbito de las AFD.

Es importante resaltar que la orientación de metas hacia la tarea se relaciona positivamente con el desarrollo de patrones de conducta adaptativos (Roberts, Kuncel, Shiner, Caspi, \& Goldberg, 2007), ajustándose al modelo de predicción que se encontró, donde valores altos de orientación a la tarea, diversión y esfuerzo pueden predecir significativamente la manifestación de motivación intrínseca en los sujetos de los tres países, correlacionando también con valores bajos de aburrimiento entre los españoles. Estas consideraciones resaltan la importancia de ser capaz de comprender e influir sobre el contexto que rodea a los jóvenes deportistas para producir niveles óptimos de motivación entre los participantes y los beneficios que de ella se derivan (Keegan et al., 2009).

Otros autores también han hallado una correlación positiva que logra predecir la motivación intrínseca a partir de la orientación hacia la tarea (Cury, Biddle, Sarrazin, \& Famose, 1997; Fox, Goudas, Biddle, Duda, \& Armstrong, 1994; Granero-Gallegos, BaenaExtremera, Pérez-Quero, Ortiz-Camacho, \& Bracho-Amador, 2012; Gutiérrez, 2014; McNeill \& Wang, 2005; Papaioannou, Bebetsos, Theodorakis, Christodoulidis, \& Kouli, 2006). Así, Papaioannou et al. (2006) encontraron que la orientación de metas predice la motivación intrínseca de los sujetos, lo cual supondrá una actitud positiva hacia la práctica de AFD gracias al desarrollo de patrones motivacionales adaptativos. Además, este estudio reflejó que niveles altos de percepción de habilidad lograron que los practicantes manifestaran motivación intrínseca hacia la práctica de AFD, si bien es cierto que la percepción de habilidad fue medida con la escala de percepción de la competencia deportiva. Por otro lado, Fox et al. (1994) hallaron que los niños con una alta orientación a la tarea estaban más motivados que aquellos con una alta orientación al ego, por lo que concluyeron que la orientación a la tarea parece ser uno de los elementos fundamentales para manifestar motivación positiva y diversión hacia la práctica de AFD.

En este estudio, también se encontró que el índice de autodeterminación puede predecirse significativamente en los tres países, obteniendo valores altos de orientación a la tarea y esfuerzo. En esta línea, Cury et al. (1997) determinaron que aquellos individuos con alta orientación a la tarea intentan superar pruebas trabajando duro y atribuyen sus éxitos a su propio esfuerzo; al mismo tiempo que atribuyen los fallos a la falta de esfuerzo y de concentración, por lo que tratan de seguir desarrollando conductas adaptativas y autodeterminadas. Para McNeill y Wang (2005), la orientación a la tarea correlaciona moderadamente con la motivación intrínseca 
y la regulación identificada, siendo estos los tipos de conductas más autodeterminados. Con una muestra de escolares británicos de entre 11 y 15 años también se demostró que aquellos con una orientación alta a la tarea mostraban una relación positiva con la manifestación de comportamientos motivacionales adaptativos, lo cual supone la presencia de conductas autodeterminadas (Wang \& Biddle, 2001). Además, esta misma investigación determinó que los sujetos con una alta amotivación mostraban una orientación a la tarea muy baja, coincidiendo con nuestros resultados y con los de GraneroGallegos et al. (2012), quienes encontraron que la amotivación correlacionaba positivamente con niveles elevados de aburrimiento y negativamente con diversión y orientación a la tarea.

Nuestros resultados también han encontrado que el índice de autodeterminación podría predecirse con niveles bajos en la orientación al ego, excepto en México; así como niveles altos de aburrimiento y de la percepción de que las técnicas de engaño son causantes del éxito en el deporte (Tabla 4). Por tanto, la orientación al ego está relacionada con la regulación externa, que es el nivel más alto de motivación extrínseca (McNeill \& Wang, 2005).

\section{Conclusiones}

La motivación intrínseca hacia la práctica físicodeportiva muestra valores más elevados en los tres países, siendo los estudiantes mexicanos los que declararon mayores niveles de motivación en la mayoría de las dimensiones analizadas.

Los tres países mostraron valores estadísticamente diferentes entre sí, respecto de Motivación en la práctica físicodeportiva, Orientación a Metas de Logro y la Percepción de las Creencias sobre las Causas del Éxito en el Deporte, tan solo en la Satisfacción Intrínseca en el Deporte, los estudiantes costarricenses manifestaron resultados diferentes y significativamente más bajos a los mexicanos y españoles. En los tres países se encontró que la Amotivación está asociada con niveles elevados de aburrimiento y niveles bajos de diversión y orientación a la tarea. El Índice de Autodeterminación puede predecirse en los tres países a partir de los valores de Orientación a la Tarea y Esfuerzo.

Estos hallazgos sugieren importantes claves para la aplicación práctica del presente trabajo, demostrando que los adolescentes que practican AFD pueden variar la percepción que tienen sobre las causas del éxito en el deporte. En este sentido, se debería promover tareas en las que las metas de logro se orienten a la tarea y hacia mejorar los niveles de motivación en los estudiantes.

\section{Limitaciones del estudio}

Una dificultad que se debe mencionar en la debilidad de un trabajo de estas características, que trata de establecer la línea base de un estudio longitudinal, es que la mortandad de la muestra suele ser grande (problema compartido en algunas de las investigaciones referidas y llevadas a cabos en otros países como Finlandia, Bélgica y Estados Unidos). Aunque el tamaño de la muestra es considerable, no se realizó un estudio de muestreo para conocer su representatividad.

\section{Perspectivas futuras}

Considerar la pertinencia de profundizar sobre el rol que desempeña el agente social de referencia (padres, amigos y el profesor de educación física), así como la influencia de las clases de educación física sobre la percepción de autoeficacia y su relación con la actitud y el comportamiento de los sujetos para la actividad física de tiempo libre.

Es necesario abordar el estudio del abandono la práctica de actividad físicodeportiva, indagando sobre el tipo de motivación con la que se inició la práctica y las causas que originaron el abandono. Así como brindar las pautas para su posible intervención. 


\section{Referencias}

Abraldes, J. A., Granero-Gallegos, A., Baena-Extremera, A., Gómez-López, M., \& Rodríguez-Suárez, N. (2016). Goal orientations, satisfaction, beliefs in sport success and motivational climate in swimmers. Revista Internacional de Medicina y Ciencias de la Actividad Fisica y del Deporte, 16(63), 583-599. http://dx.doi.org/10.1536 6/rimcafd2016.63.011

Balaguer, I., Atienza, F. L., Castillo, I., Moreno-Murcia, J. A., \& Duda, J. (septiembre, 1997). Factorial structure of measures of satisfaction/interest in sport and classroom in the case of Spanish adolescents. Trabajo presentado en la IV European Conference of Psychological Assessment, Lisboa, Portugal.

Balaguer, I., Castillo, I., \& Tomás, I. (1996). Análisis de las propiedades psicométricas del cuestionario de orientación al Ego y a la Tarea en el deporte (TEOSQ) en su traducción al castellano. Psicológica, 17, 71-81. https://www.uv.es/icastill/document os/1996.BalaguerCastilloTomas96_Psicolo gica.pdf

Böckler, A., Tusche, A., \& Singer, T. (2016). The structure of human prosociality: Differentiating altruistically motivated, norm motivated, strategically motivated, and self-reported prosocial behavior. Social Psychological and Personality Science, 7(6), 530-541. http://dx.doi.org/10.1177/194855 0616639650

Bollen, K. A., \& Long, J. S. (1994). Testing structural equation models. Newbury Park, CA: Sage.

Brooks, J. M., Iwanaga, K., Chiu, C. Y., Cotton, B. P., Deiches, J., Morrison, B., . . . Chan, F. (2017). Relationships between self-determination theory and theory of planned behavior applied to physical activity and exercise behavior in chronic pain. Psychology, Health and Medicine, 1-9. http://dx.doi.org/10.1080/13 548506.2017.1282161
Carretero-Dios, H., \& Pérez, C. (2005). Normas para el desarrollo y revisión de estudios instrumentales. International Journal of Clinical and Health Psychology, 5(3), 521-551. Recuperado de http://www. redalyc.org/html/337/33705307/

Castillo, I., Balaguer, I., \& Duda, J. L. (2002). Las perspectivas de meta de los adolescentes en el contexto deportivo. Psicothema, 14(2), 280-287. Recuperado de https://www.uv.es/icastill/documentos/2 002.CastilloBalaguerDuda02_Psicothema. pdf

Cury, F., Biddle, S., Sarrazin, P., \& Famose, J. P. (1997). Achievement goals and perceived ability predict investment in learning a sport task. Br J Educ Psychol, 67 ( Pt 3), 293-309; discussion 339-243.

da Costa, C. E., Hirota, V. B., \& de Marco, A. (2015). Motivational goals orientation in Physical Education classes of elementary education. Journal of Physical Education and Sport, 15(2), 167-171. http://dx.doi.org/10. 7752/jpes.2015.02026

Deci, E. L., \& Ryan, R. M. (1985). Intrinsic motivation and self-determination in human behavior. New York: Plenum.

Deci, E. L., \& Ryan, R. M. (2000). The "what" and "why" of goal pursuits: Human needs and the self-determination of behaviour. Psychological Inquiry, 11(4), 227-268.

Duda, J. L. (1989). Relationship between task and ego orientation and the perceived purpose of sport among high school athletes. Journal of Sport \&ु Exercise Psychology, 11(3), 318-335.

Duda, J. L., \& Nicholls, J. G. (1992). Dimensions of achievement motivation in schoolwork and sport. Journal of Educational Psychology, 84(3), 290-299.

Duda, J. L., \& Whitehead, J. (1998). Measurement of goal perspectives in the physical domain Advances in sport and exercise psychology measurement (pp. 21-48). Morgantown, WV: Fitness Information Technology.

Fletcher, J. (2016). Applying SelfDetermination Theory to College 
Students' Physical-Activity Behavior: Understanding the Motivators for Physical (In) Activity. Communication Studies, 67(5), 489-508. http://dx.doi.org/10.1080/105109 74.2016.1212911

Fox, K., Goudas, M., Biddle, S., Duda, J., \& Armstrong, N. (1994). Children's task and ego goal profiles in sport. Br J Educ Psychol, 64 ( Pt 2), 253-261.

Franco, E., Coteron, J., Gómez, V., Brito, J., \& Martinez González, H. A. (2017). Influencia de la motivación y del flow disposicional sobre la intención de realizar actividad físico-deportiva en adolescentes de cuatro países. Retos. Nuevas Tendencias en Educación Física, Deporte y Recreación, 31, 46-51. Recuperado de https://recyt.fecyt.es /index.php/retos/article/view/39713/31168

Franco, E., Coterón, J., Martínez, H. A., \& Brito, J. (2017). Perfiles motivacionales en estudiantes de educación física de tres países y su relación con la actividad física. Suma Psicológica, 24(1), 1-8. http://dx.doi.o $\mathrm{rg} / 10.1016 / \mathrm{j}$.sumpsi.2016.07.001

Granero-Gallegos, A., Baena-Extremera, A., Pérez-Quero, F. J., Ortiz-Camacho, M. M., \& Bracho-Amador, C. (2012). Analysis of motivational profiles of satisfaction and importance of physical education in high school adolescents. Journal of Sports Science and Medicine, 11(4), 614-623. Recuperado de http:// europepmc.org/articles/PMC3763306

Grastén, А. (2016). Testing the model of motivational climate, goal orientations, expectancy beliefs, task values in school physical education, and associated links to light- to vigorous-intensity physical activity. International Journal of Sport Psychology, 47(5), 408-427. http://dx.doi.org/10.7352/I JSP-2016.47.408

Gutiérrez, M. (2014). Relaciones entre el clima motivacional, las experiencias en educación física y la motivación intrínseca de los alumnos. Retos. Nuevas Tendencias en Educación Física, Deporte y Recreación, 26, 9-14. Recuperado de https://recyt.fecyt.es/i ndex.php/retos/article/view/34387
Gutiérrez, M., Tomás, J. M., \& Calatayud, P. (2017). Influencia del clima motivacional en educación física sobre las metas de logro y la satisfacción con la vida de los adolescentes. Retos. Nuevas Tendencias en Educación Física, Deporte y Recreación, 31, 157-163. Recuperado de https:/recyt.fecyt.es/index.php/retos/art icle/view/49421/32144

Han, D., Mahony, D. F., \& Greenwell, T. C. (2016). A comparative analysis of cultural value orientations for understanding sport fan motivations. International Journal of Sports Marketing and Sponsorship, 17(3), 260-276. http://dx.doi.org/10.1108/IJSMS08-2016-016

Hoyle, R. H. (1995). Structural equation modeling: Concepts, issues, and applications. Thousand Oaks, CA: Sage.

Hu, L., \& Bentler, P. M. (1999). Cutoff criteria for fit indexes in covariance structure analysis: Conventional criteria versus new alternative. Structural Equation Modeling: A Multidisciplinary Journal, 6(1), 1-55. http:// dx.doi.org/10.1080/10705519909540118

Jaakkola, T., Ntoumanis, N., \& Liukkonen, J. (2016). Motivational climate, goal orientation, perceived sport ability, and enjoyment within Finnish junior ice hockey players. Scandinavian Journal of Medicine and Science in Sports, 26(1), 109-115. http://dx. doi.org/10.1111/sms.12410

Keegan, R., Harwood, C., Spray, C., \& Lavallee, D. (2009). A qualitative investigation exploring the motivational climate in early career sports participants: Coach, parent and peer influences on sport motivation. Psychology of Sport and Exercise, 10(3), 361-372. http://dx.doi.org/10.1016/j.psychs port.2008.12.003

Keegan, R., Middleton, G., Henderson, H., \& Girling, M. (2016). Auditing the socioenvironmental determinants of motivation towards physical activity or sedentariness in work-aged adults: A qualitative study. BMC Public Health, 16(1). http://dx.doi.org/10.1 186/s12889-016-3098-6 
Luis A. Berlanga, Constanza Palomino Devia, Francisco Ruiz-Juan, et al.

Kim, B. J., Williams, L., \& Gill, D. L. (2003). A cross-cultural study of achievement orientation and intrinsic motivation in young USA and Korean athletes. International Journal of Sport Psychology, 34(2), 168-184.

Kline, R. B. (1998). Principle and practice of structural equation modeling. Nueva York: Guilford.

Kouli, O., \& Papaioannou, A. G. (2009). Ethnic/ cultural identity salience, achievement goals and motivational climate in multicultural physical education classes. Psychology of Sport and Exercise, 10(1), 45-51. http://dx.doi.org/10.1016/j.psychspo rt.2008.06.001

Locke, E. A., \& Bryan, J. F. (1969). The directing function of goals in task performance. Organizational Behavior and Human Performance, 4(1), 35-42. http://dx. doi.org/10.1016/0030-5073(69)90030-0

McNeill, M. C., \& Wang, C. K. J. (2005). Psychological profiles of elite school sports players in Singapore. Psychology of Sport and Exercise, 6(1), 117-128. http://dx.doi.org/1 0.1016/j.psychsport.2003.10.004

Menéndez Santurio, J. I., \& Fernández-Río, J. (2017). Responsabilidad social, necesidades psicológicas básicas, motivación intrínseca y metas de amistad en educación física. Retos. Nuevas Tendencias en Educación Física, Deporte y Recreación, 32, 134-139. Recuperado de https://recyt.fecyt.es/index. php/retos/article/view/52385/33653

Moreno-Murcia, J. A., \& Martínez, A. (2006). Importancia de la Teoría de la Autodeterminación en la práctica físico-deportiva. Cuadernos de Psicología del Deporte, 6(2), 39-54. Recuperado de http://revistas.um.es/cpd/article/view/11 $3871 / 107881$

Morgan, K. (2017). Reconceptualizing motivational climate in physical education and sport coaching: An interdisciplinary perspective. Quest, 69(1), 95-112. http://dx .doi.org/10.1080/00336297.2016.1152984

Murray, E. J. (1964). Motivation and emotion. New Jersey: Prentice Hall.
Nicholls, J. G. (1989). The competitive ethos and democratic education. Cambridge, MA: Harvard University Press.

Nishida, T., Isogai, H., Åström, P., Karp, S., \& Johansson, M. (2007). Crosscultural comparison of motivation to learn in physical education: Japanese vs Swedish schoolchildren. Psychological Reports, 101(2), 597-613. http://dx.doi.org/ 10.2466/PR0.101.2.597-613

Núñez, J. L., Martín-Albo, J., Navarro, J. G., \& González, V. M. (2006). Preliminary validation of a Spanish version of the sport motivation scale. Perceptual and Motor Skills, 102(3), 919-930. http://dx.doi.org/10 $.2466 /$ pms.102.3.919-930

O'Doherty, J. P. (2016). Multiple systems for the motivational control of behavior and associated neural substrates in humans. En M. A. Geyer, B. A. Ellenbroek, C. A. Marsden, T. R. E. Barnes \& S. L. Andersen (Eds. de la Serie) \& H. E. Simpson (Ed. del Volumen), The behavioral neuroscience of motivation: Vol 27. Current Topics in Behavioral Neurosciences (pp. 291-312). Ginebra: Springer International Publishing. Papaioannou, A., Bebetsos, E., Theodorakis, Y., Christodoulidis, T., \& Kouli, O. (2006). Causal relationships of sport and exercise involvement with goal orientations, perceived competence and intrinsic motivation in physical education: a longitudinal study. Journal of Sports Sciences, 24(4), 367-382. http://dx.doi.org/ 10.1080/02640410400022060

Pelletier, L. G., Fortier, M. S., Vallerand, R. J., \& Tuson, K. M. (1995). Toward a new measure of intrinsic motivation, extrinsic motivation, and amotivation in sports: The Sport Motivation Scale (SMS). Journal of Sport E Exercise Psychology, 17(1), 35-53. h $\mathrm{ttp} / / /$ dx.doi.org/10.1123/jsep.17.1.35

Robbins, S. P., \& Judge, T. A. (2009). Comportamiento organizacional. México: Pearson Educación.

Roberts, G. (2012). Motivation in sport and exercise from an achievement goal theory perspective: After 30 years, where are 
we? En G. Roberts \& D. Treasure (Eds.), Advances in motivation in sport and exercise (3a. ed., pp. 5-58). Champaign, IL: Human Kinetics.

Roberts, B. W., Kuncel, N. R., Shiner, R., Caspi, A., \& Goldberg, L. R. (2007). The power of personality: The comparative validity of personality traits, socioeconomic status, and cognitive ability for predicting important life outcomes. Perspectives on Psychological Science, 2(4), 313-345. http://dx.doi.org/10.1111/j.17456916.2007.00047.x

Ryan, R. M., \& Deci, E. L. (2000). Selfdetermination theory and the facilitation of intrinsic motivation, social development, and well-being. American Psychology Association, 55(1), 68-78. http://dx.doi.org/ 10.1037110003-066X.55.1.68

Salguero, A., Gonzalez-Boto, R., Tuero, C., \& Marquez, S. (2003). Identification of dropout reasons in young competitive swimmers. The Journal of Sports, Medicine, and Physical Fitness, 43(4), 530-534. Recuperado de https://www.researchgate.n et/publication/8883127

Smith, R. E., Cumming, S. P., \& Smoll, F. L. (2008). Development and validation of the Motivational Climate Scale for Youth Sports. Journal of Applied Sport Psychology, 20(1), 116-136. http://dx.doi.org/10.1080/ 10413200701790558

Stuntz, C. P., \& Weiss, M. R. (2009). Achievement goal orientations and motivational outcomes in youth sport: The role of social orientations. Psychology of Sport and Exercise, 10 (2), 255-262. http://dx .doi.org/10.1016/j.psychsport.2008.09.001

Vallerand, R. J. (1997). Toward a hierarchical model of intrinsic and extrinsic motivation. En M. P. Zanna (Ed.), Advances in Experimental Social Psychology (Vol. 29, pp. 271-360). Nueva York/San Diego: Academic Press.

Vallerand, R. J. (2012). Intrinsic and extrinsic motivation in sport and physical activity: A review and a look at the future. En G. Tenenbaum \& R. C. Eklund (Eds.),
Handbook of sport psychology (3a. ed., pp. 59-83). Nueva York: Wiley.

Vallerand, R. J., \& Rousseau, F. R. (2001). Intrinsic and extrinsic motivation in sport and exercise: A review using the hierarchical model of intrinsic and extrinsic motivation. En R. S. H. Hausenblas \& C. Janelle (Eds.), Handbook of sport psychology (2a. ed.). Nueva York: Wiley.

Vilchez Conesa, M. P., \& Ruiz Juan, F. (2016). Clima motivacional en Educación Física y actividad físico-deportiva en el tiempo libre en alumnado de España, Costa Rica y México. Retos. Nuevas Tendencias en Educación Física, Deporte y Recreación, 29, 195-200. Recuperado de https://recyt.fecyt.es/index.php/retos/art icle/view/42448/25471

Vink, K., Raudsepp, L., \& Kais, K. (2015). Intrinsic motivation and individual deliberate practice are reciprocally related: Evidence from a longitudinal study of adolescent team sport athletes. Psychology of Sport and Exercise, 16(P3), 1-6. http://dx.doi.org/10.1016/ j.psychsport.2014.08.012

Vroom, V. H. (1964). Work and motivation. NuevaYork: Wiley.

Wang, C. K., \& Biddle, S. (2001). Young people's motivational profiles in physical activity: A cluster analysis. Journal of Sport $\mathcal{E}$ Exercise Psychology, 23(1), 1-22. http://dx.doi.org/0. 1123/jsep.23.1.1

Yan, J. H., \& McCullagh, P. (2004). Cultural influence on youth's motivation of participation in physical activity. Journal of Sport Behavior, 27(4), 378-390. Recuperado de https://www.highbeam.com/doc/1G1-12 5568949.html

Zaff, J. F., Moore, K. A., Papillo, A. R., \& Williams, S. (2003). Implications of extracurricular activity participation during adolescence on positive outcomes. Journal of Adolescent Research, 18(6), 599-630. http ://dx.doi.org/10.1177/0743558403254779

Zahariadis, P. N., \& Biddle, S. J. H. (febrero, 2000). Goal orientations and participation motives in physical education 
Luis A. Berlanga, Constanza Palomino Devia, Francisco Ruiz-Juan, et al.

and sport: Their relationships in English schoolchildren. Athletic Insight. The Online Journal of Sport Psychology, 2(1), 1-12. http: //dx.doi.org/10.1.1.537.3984

\section{Notas}

* Artículo de investigación. 